\title{
DOCUMENTATION
}

\section{Notes sur la Construction des Premiers Transformateurs à 220.000 volts.}

\section{I. - Considérations générales.}

L'intérêt du "Superpower system ", qui était déjà apparu aux techniciens avertis, bien avant la guerre, s'est considérablement accentué, pendant et depuis la tourmente, par le fait du bouleversement général, des crises industrielles qui en ont découlé, dü ralenlissement constaté dans la production des charbons et huiles lourdes, enfin de la paralysie générale qui a atteint les chemins le fer clu monde entier et de l'intensification de leur électrificalion, envisagée partout comme le spécifiçue de cette paralysie.

Techniciens et compagnies ont, depuis longtemps du reste, prévi la nécessité de réaliser pour les transports de force, des voltages heaucoup plus élevés que ceux adoptés jusqu'à ce jour, en même (emps que tes réseaux plus étendus et mietıx reliés entre eux, de facon à diminuer les pertes de puissance - qui sont, à l'heure acluelle, relativement considérables - et assurer, en même temps qu'une répartition plus judicieuse, une utilisation plus rationnelle the l'énergie du pays, susceptible de libérer les chemins de ler, (lt) même qu'une notable partie de l'Industrie, de leurs onéreux transports de charbons.

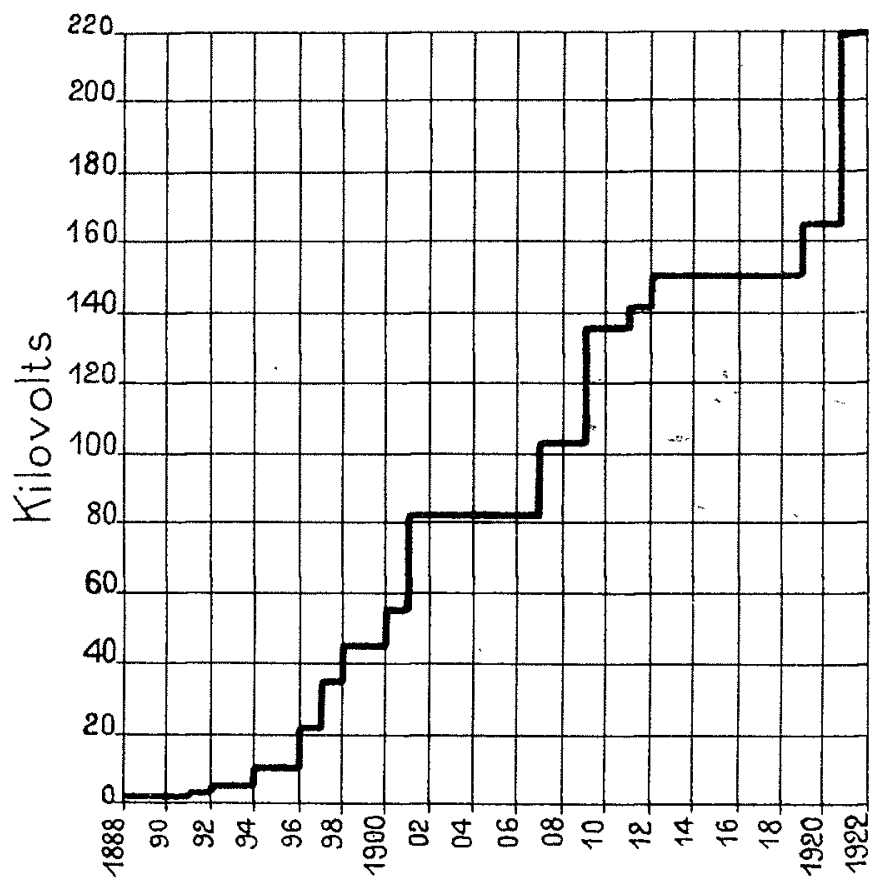

Années

lïg. 1. - Graphique statistique résumant les progrès successifs realisés dans la tension des transformateurs pour transports de force.

I a tension de 200 à 220.000 volts a généralement été reconnuc comme étant celle de plus grande économie pour ces extensions el interconnections. Jusqu'à sa première réalisation elle a effrayé bien des gens et trouvé bien des sceptiques ; aujourd'hui la plupart sont convertis, et malgré que l'étape franchie par cette réalisation dans l'accroissement constant des tensions de transport (fig. 1) soit considérable par rapport aux précédentes, il semble désormais certain que non seulement l'emploi de 220.000 volts aura le même succès que celui qui souligna, peu avant la guerre, l'apparition du premier appareil à 150.000 volts de la "Southern California Edison Co ", mais qu'on ne s'en tiendra pas là...

Ce pas brutal avait, du reste, été fort discuté, en 1919 et 1920 , par de nombreuses sommités techniques, avant d'être franchi, et l'on peut qualifier d'hérol̈que la décision que prit, à l'automne clernier, cette même compagnie "S. C. E. " de clore la controverse en commanclant ferme à la "General Electric $C^{0}$ " 4 unités élévatrices à ce potentiel : $11.000 / 220.000 \mathrm{~V} .-50 \sim$ d'une puissance de 8.3:33 KVA, destinces à être annexées à sa nouvelle centrala hydro-électrique de "Big Creek $N^{\circ} \&$ " et à constituer, sur un parcours de $385 \mathrm{Kms}$, la première artère d'un réseau considérable, transportant et répartissant $750.000 \mathrm{HP}$, à $220.000 \mathrm{~V}$. dans la région de Los Angeles (Californie).

Aucun délai n'a ćté fixé pour la livraison de ces appareils, mais Lout a été mis en œuvre pour qu'il soit aussi réduit que possible, car les lignes actuelles de la "S.C. E. Co" sont chargées au maximum et il est indispensable d'augmenter considérablement la puissance mise à la disposition de la Province, dont toutes les centrales doivent être reliées par le réseau T. H. T. à $220.000 \mathrm{~V}$. visé plus haut.

En fait, l'origine de la construction en bobine clans les transformateurs à haut voltage remonte aux premiers essais effectués par la "G. E. $C^{\circ}$ ", avec des potentiels de 70 à $100 \mathrm{KV}$; ensuite descuels, cette puissante Compagnie entreprit une vaste enquête de longue haleine, complétée par de multiples expériences de laboratoire. et destinée à préciser toutes les données du problème dont certaines étaient encore mal établies. A cette enquête, la "Colorado Power Co " et la "Great Western Power Co " équipées de date récente à $110.000 \mathrm{~V}$, fournirent d'importants tributs qui, joints à la transposition dans le laboratoire - au moyen d'appareils de construction spéciale - des phénomènes de l'exploitation normale, permirent d'expliquer, en même temps que de résoudre nombre de difficultés qui semblaient s'opposer pratiquement à de nouveaux accroissements de tension, et de démontrer que le problème qui se posait, pour passer de 100.000 à $220.000 \mathrm{~V}$, quoique d'apparence plus sévère, était, en réalité; de résolution plus facile, une fois ces données bien établies, que celui qui se posa antérieurement pour passer de 70.000 à $100.000 \mathrm{~V}$.

L'enquête et les études - fort longues et onéreuses - de la "G. E. Co " constituent à la fois, un des plus beaux titres de gloire de cette Société, et l'un des monuments d'expérimentation les plus importants de la Science électrotechnique qui aient vu le jour dans le courant des douze dernières années.

\section{II. - Avantages des bobines circulaires pour les transformateurs à hauts voltages.}

De longue date déjà, la "G. E. Co "emploie la bohine circulaire pour la construction de ses transformateurs de toutes puissances. L'expérience acquise par cette Compagnie, tant dans les premiers développements des unités de grandes puissances - alors que la cage à enroulements rectangulaires était universellement employée - que pendant ces dernières ammées, qui ont vu tant de progrès remarquables en capacité aussi bien qu'en voltage, soulignée par les résultats de haute valeur pratique qu'elle a dès longtemps obtenus, démontre surabondamment que l'enroulement circulaire est le seul qui convienne aux très hautes tensions et que les dangers menaçant encore l'extension des puissances et des volges sont inhérents, pour la plus grande partie, à quelque particularité constructive dépendant entièrement de la forme de la bobine.

D'autre part, les expériences effectuées à l'Institut électrotechnique de Schenectady (G. E. C.) ayant ćtabli que sous l'influence des sollicitations induites par le champ magnétique les bobines de formes diverses subissent invariablement une déformation les rapprochant de la forme eirculaire, alors que celles ayant déjà cette forme résistent beaucoup plus allègrement à ces sollicitations, la supériorité mécanique de la bobine circulaire en pré- 
sence des effets destructifs du court-circuit découle inmécliatement de ce lait; en outre de ce que, au point de vue constructif, la forme circulaire rend plus facile le soutien des conducteurs à intervalles très courts, par supports en croix, sans entraver la circulation de Thuile de refroidissement - disposition qui permet de réduire l'échauffement des organes de soutien des bobines gràce à une plus grande efficacité du refroidissement obtenue en faisant circuler l'huile à la fois dans la direction des conducteurs et perpendiculairement à cette direction.

Le premier avantage est encore accru par l'usage d'enroulements très étroits et de supports-espaceurs de groupes à angle droit avec les conducteurs, réduisant ainsi à quelques millimètres la longueur du chenal conducteur de chaleur entre l'axe des supports et l'huile de refroidissement. Le second résulte de l'agencement de l'enroulement circulaire où l'huile coule en travers, et dont la faible longueur du chenal d'huile est déterminée par la largeur de l'enroulement plutôt que par sa longueur.

Combinés, ces résultats ont rendu possible la construction d'unités de grande puissance à potentiels élevés dans lesquelles la température du "point chaud " est dûe principalement à la différence entre les températures de l'huile au sommet et à la base du caisson.

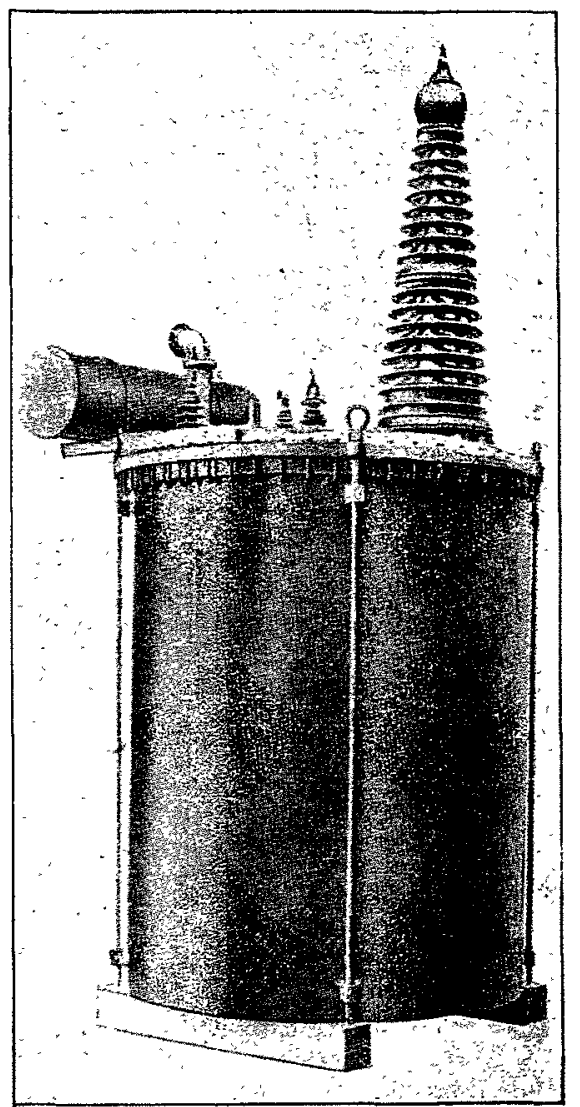

Jïg. 2. - Vue générale du nouveau transformateur de 8.393 K.V.A. a $11.000 / 220.000$ V. $-50 \sim$ de la "Southern California Edison $C^{\circ} \mathrm{B}$.

\section{III. - Protection de lisolement des bobines contre les détériorations.}

La forme circulaire des bobines facilite également la protection de l'isolement - partie vitale de toute construction - dont elle augmente l'efficacité contre les éventuels dérangements d'ordre mécanique.

Cet isolement dont l'encombrement est très restreint, et qui doit néanmoins pouvoir supporter de très hauts voltages localisés, est toujours constitué par un grand nombre de minces couches de matière isolante très subtile, dont la conservation est assurée par la forme circulaire de l'enroulement, à courbure régulière, sans angles saillants ni pointes, et qui se contracte ou se dilate uniformément suivant les variations de température. Et comme avec celle de l'isolement, augmente, parallèlement, l'importance des rétériorations possibles, et, par voie de conséquence, celle de sa protection, un nouvel avantage des bobines circulaires intervient encore avec leur indéformabilité, qui évite toute obstruction des concluits de circulation d'huile pouvant provenir de depots dans l'huile ou de déformations dans les enroulements el occasionner un court-circuit entre les bobines a premiere surtension dans la ligne.

\section{IV. - Les nouvelles unités $11.000 / 220.000 \mathrm{~V}$. de la "S. C. E. C" ".}

Ces nouveaux transformateurs - les premiers a parcille tension (fig. 2) - sont, dans leur ensemble, très proches parents de ceux ì $165.000 \mathrm{~V}$. (7.500 Kva) de la "Great Western Hower (\%". Comme ces derniers, ils comportent, sur deux noyaux d'armature pour chaque phase, deux grompes d'enroulements du type concentrique, avec isolements assurés par un certain nombre de cylindres espacés et assujettis de manière à ne pas entraver la circulation de l'huile (Fig. 3), et sont construits pour une mise a la terre permanente du point neutre en cours de service. M. Clinton Jones en donnail dernièrement une description détaillée dans l'Electrical Worlel. d'où sont extraites les caractéristiques résumées ci-après (1).

l'extrémité de l'enroulement à hate tension sort à lravers un isolateur du nouveau type règlementaire à manchon rempli d'huile

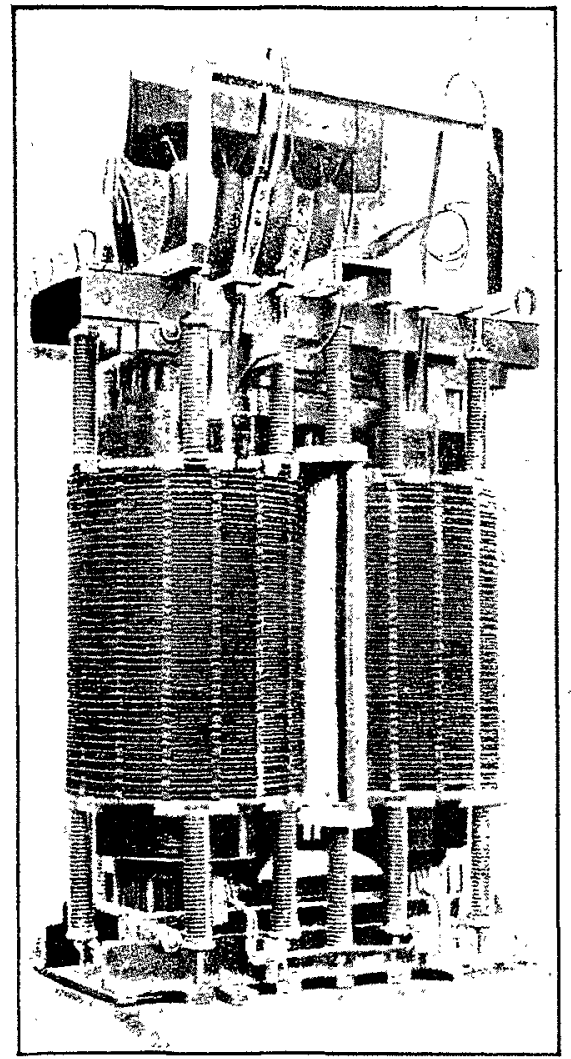

Fig. 3. - Montage intérieur, enroulements et isolements, des nouveaux transformateurs à $220,000 \mathrm{~V}$. le la "S. C. E. Co".

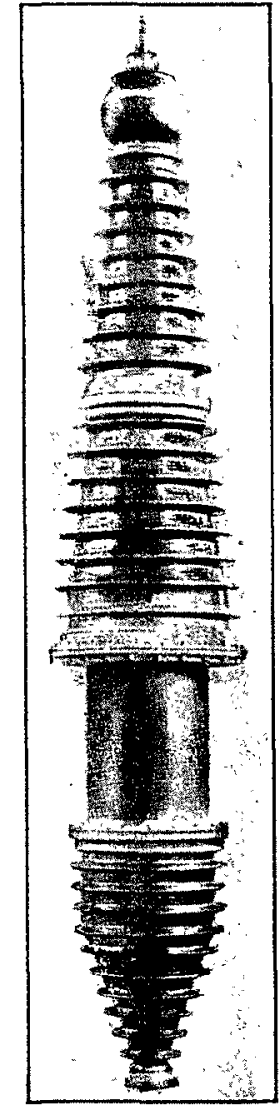

Fig. 1. - Isolateur métal-porcelaine des nouveaux transtormateurs à $220.000 \mathrm{~V}$. a 250.000 V., interchangeable tant entre les transformateurs qu'avec les disjoncteurs. La carcasse de cet isolateur (Fig 1) comporte trois pièces de porcelaine dont deux à la partie supéricure, au-dessus du couvercle du caisson, et une à l'extrémité inféricure, réunies entre elles par un manchon métallique descendant invariablement en dessous du niveau de l'huile. Un tube de mctal servant de guide au câble conducteur, et situé au centre de sa section transversale, traverse cette carcasse du haut en bas avec interposition, par alternances, entre carcasse et tube, de couches cylindriques d'isolant et d'espaces pleins d'huile. I,es joints entre les différentes parties de l'isolateur sont.assurés par des couronnes en liège comprimées par de nombreux boulons, et une chambre sphérique en verre, située au-dessus, sert à la fois pour l'expansion de l'huile et pour l'indication permanente de son niveau.

(1) Electrical World, Vol. 77, No 6. Mc Graw Hill co, edilor, New-York, 1921 
L'isolateur est construit pour une tension de décharge superficielle à sec de 660.000 volts, et de telle façon qu'une répartition superficielle uniforme du polentiel permette d'éviter le phénomènecouronne.

I.e caisson est du nouveau type à conservateur d'huile, comportant une chambre séparéc pour l'expansion de l'huile (Fig. 2). Ainsi que nous l'avons exposé dans une précédente étude publiée tout réccmment par un de nos confrères (1), le principe du conservateur d'huile réside dans l'élimination de la couche d'air régnant habituellement dans le caisson entre son couvercle et le niveau de l'huile (qu'il imprègne d'humidité), par l'adjonction d'un réservoir auxiliaire dit " conservateur " situé au-dessus du caisson principal, communiquant avec lui par un tube et servant à l'expansion de l'huile dont ce dernier est complètement rempli ainsi que le tube de communication : le danger de pénétration de l'huile sous pression est évité par l'adjonction au conservateur d'un reniflard plaré sur la chambre d'expansion, laquelle est, en outre, munie, à sa parlie inlérieure, d'un robinel de purge pour l'evacuation de l'ean de condensation et des dépôts éventuels.

Avec ce dispositil, le caisson est maintenu constamment plein par le conservateur au moyen du tube de communication ; ainsi isolée, l'huile y demeure parfaitement sèche et limpide, et les gaz. les décompositions dûes à de trop fortes surtensions ne pouvant s'y mélanger à l'air pour former des hydrocarbures, tout danger d'explosion se trouve écarté de ce fail (2).

Le transformateur de courant monté sur le couvercle est connecté avec le neutre en amont de sa mise à la terre, et ceux des trois

(1) Voir La Vie Tcchnique et Industrielle de juin 1922 : "Les Nouveaux caissons à conservateur d'huile pour transformateurs".

(2) Du fait de l'intercommunication des réservoirs, les gaz pouvant être emprisonnés dans l'huile se rendent automatiquement dans le conservateur où manquent complètement, pour eux, les possibilités d'ignition existant dans le caisson principal. phases ont leur secondaire en parallèle de façon que soit identifié tout courant vers la terre ou déséquilibre dans la charge des phases.

Le poids total de ces nouvelles unités y compris l'huile de remplissage atteint 50 tonnes (1) ; leur diamètre est de $3 \mathrm{~m}$. 20 et leur hauteur totale entre le rail et le sommet de l'isolateur, de $7 \mathrm{~m}$. 30 . C'est assez souligner l'importance de ces monuments et le progrès décisif cu'ils représentent par rapport à ceux en service au voltage maximum actuellement réalisé, qui est, croyons-nous, de $165.000 \mathrm{~V}$.

A l'heure où nous écrivons ces lignes, les nouveaux transformateurs à $220.000 \mathrm{~V}$. construits par la " $G$. E. $C^{\circ}$ " doìvent être entrés en service; nous ne manquerons pas de donner les caractéristiques de leurs épreuves clès que nous en connaîtrons les résultats, mais d'ores et déjà nous pouvons signaler que la "S. C. E. Co" ne les a pas même attendus pour commander par ailleurs de nouvelles unités à semblable tension : c'est dire toute la confiance qu'on a, chez nos amis d'Amérique, non seulement en ces appareils spéciaux, aujourd'hui entrés dans le domaine de la pratique, mais surtout dans la possibilité d'énormes accroissements des voltages de transport hier encore considérés comme indépassables (2). Souhaitons que cette belle confiance pénètre également dans nos milieux techniques, et qu'instruits par l'expérience ces derniers sachent en tirer tout le profit possible : les grands projets d'aménagements qui mùrissent en France, pendant qu'on réalise à côté, ne sauront qu'y gagner.

J. B.

(1) La tome anglaise vaut 1016 kilogs.

(2) Signalons, à ce sujet, les récentes expériences elfectuées dans les laboratoires de Ia "G. E. $C^{\circ}$ " sur un transformateur d'épreuve de $1000 \mathrm{KVA}$ alimenté à $2.300 \mathrm{~V} .-60 \sim$, dont la tension secondaire fut élevée à 700,900 et $1100 \mathrm{KV}$, avec des chaînes d'isolateurs comportant jusqu'à 22 éléments, et qui donnèrent des résultats si encourageants que la Compagnie envisage, dès maintenant, la réalisation pratique de tensions d'un million de volts (Voir à ce sujet la Gen. El. Rev. de lévrier dernier).

\section{Chauffage au gaz naturel de la Centrale de Midway.}

Dans cette étude, abondamment documentée, lauteur donne d'abord une description assez détaillée de la Centraje qui alimente l'industrieuse vallée de San-Joaquim et la région de Presco (Californie) pour $75.000 \mathrm{HP}$ devenus insuffisants en 1920 : il décrit ensuitches agrandissements et extensions auxquels il fallut recourir depuis cette époque pour salistaire la demande croissante de consommation. Enfin, il insiste sur la caractéristique saillante des installations qui est dans le chauffage aux gaz naturels, au moyen de dispositils dont il donne également la description, et cui permettent d'amener aux foyers les gaz, captésì 11 kilomètres de l'usine, au moyen de pompes, à une pression voisine de $35 \mathrm{~kg} / \mathrm{cm} 2$.

La capacité de production de cette usine est de $90.000 \mathrm{kgs}$ de vapeur par minute. Les habitations du personnel sonl également chauffées aux gaz naturels.

Electrical Reviem, 18 aoùt 1922 .

J. B.

\section{Un nouveau type de barrage en maçonnerie.}

Le type classique du barrage à gravité est certes celui qui inspire le plus de confiance, parce que possédant à son actif les plus belles références aux hauteurs maxima abordées à ce jour. Il présente, toutefois, un profil peu économique, et de différents còtés (depuis la guerre en particulier) les techniciens se préoccupent de lui substituer des ouvrages moins onéreux, quoique rarement aussi éprouvés.

Un ingénieur ilalien, M. Norzi, a été conduit à envisager comme variante du type à gravité un type d'ouvrage, pour le moins curieux, dont le parement aval est sensiblement celui du premier, mais clont le parement amont est disposé en forme de $V$ ouvert sur l'amont dont les deux tiers supérieurs sont en surplomb sur le liers inférieur. Le calcul montre malgré cette forme bizarre que la stabilité et la résistance sont en tous points comparables arec celles du type à gravité plein, alors que le cube est inférieur de $22 \%$ à la hauteur de $20 \mathrm{~m}$. seulement (1).

Industria, 15 juillet 1922.

(1) Au sujet des types de barrages économiques, nous croyons opportun de signaler à nos lecteurs l'átude très documentée de notre collaborateur M. J. Boudet, sur "Les Barrages a gravité creux ", qui va paraître prochainement dans la "VII ".

\section{Le comportement des moteurs de traction surchargés.}

En. présence de la sensibilité aux surcharges, très inégale suivant le mode de traction qu'ils assurent, ainsi que suivant leur construction, des moteurs de traction ferroviaire, lauteur de cette note souligne qu'un moyen eflicace d'éviter les surcharges est la systématisation sur la plus grande échelle possible, du convoi à unités multiples : mais qu'en pratique on n'applique pas assez son principe excellent, et que l'attelage des remorcues aux antomotrices détruit fâcheusement l'équilibre entre les éléments moteurs. Eludiant ensuite, de facon détaillee, l'efiet des surcharges de différentes durées sur les moteurs, il conclut qu'il y a lieu de $s^{\prime}$ astreindre à ne pas imposer aux moteurs ventilćs ou autoventilés, les surcharges qui seraient admissibles arec le type non ventile. cui, par rapport à leur charge normale, ont une capacité de surcharge très supérieure aux premiers.

Electrical Railway Journal, 26 août 1922.

J. $B$. 


\section{La commande à distance de la sous-station de Maidstone.}

Description d'autant plus intéressante qu'elle est laite d'après les indications de la Direction de la Compagnie, d'une des premières installations anglaises commandées à dislance par les procédés déjả couramment employés en Amérique.

La sous-station en question comporte une commutatrice de 500 Kil à $500 \mathrm{~V}$. tournant à 1.000 t.p.m. Elle est commandée d'un tableau à 5 panneaux, dont quatre fonctionnent comme panneaux le feeders, par le personnel de l'usine génératrice qui la met en charge le matin par les moyens habituels, suit sur un ampèremètre spécial les variations de la charge, el l'arrête le soir à l'aide d'un càble-guide actionnant les disjoncteurs de la commutatrice, tant du còté alternalil que du cóté continu.

L'installation fonctionne ainsi depuis quatre mois de façon très satisfaisante.

Electrical Reniew, s septembre 1922.

\section{Nouveaux fours " Héroult" de 40 tonnes !}

Le ministère de la marine américain vient de mettre en service deux nouveaux fours Héroult de chacun $40 \mathrm{~T}$. de capacité destinés a fonctionner en parallelle avec deux fours Martin de $75 \mathrm{~T}$. pour le travail de l'acier nécessaire aux pièces maxima des blindages et canons.

Après séparation du phosphore, le métal est passé aux lours électriques, où il est séparé du soufre et des acides puis porté à la température et additionné des matériaux d'alliages préalablement

\section{Synchronisation due à la capacitance}

Dans deux installations hydroélectriques on constatait à quelques mois de distance une même tendance à la synchronisation d'alternateurs alimentant séparément des lignes aériennes placées sur supports communs. Mais tandis que pour l'installation d'Inawashiro-Tokyo les alternateurs tendaient à se synchroniser en concordance de phase, à la station de San-Bernardino (Californie) on rencontrait au contraire une certaine difficulté pour le couplage en parallèle des alternateurs qui tendaient à se placer d'eux-mêmes en opposition de phase.

Il s'agissait de lignes doubles triphasẻes, et les phénomènes d'autosynchronisation constatés étaient dus aux échanges d'énergie par les capacitances.

Mais la disposition des conducteurs n'étanl pas la même dans les deux cas, on avait :

\section{Les travaux d'électrification qui seront exécutés}

Le programme prévu comporte :

270 millions de dépenses pour l'électrification ; 775 millions pour les autres travaux.

\section{RÉSEAU DU MrDI}

C'est le programme du réseau du Midi qui comporte la plus forte dépense : 124 millions.

Il comprend, tout d'abord, l'appropriation en vue des nouvelles natures de courant utilisées, des usines de Soulom et d'Eget, qui ont été construites, antérieurement, à l'occasion de lignes nouvelles.

L'aménagement de la vallée d'Ossau, comprenant la construction des usines pour la production de l'énergie, est prévu pour 30 millions, en 1923. La construction du réseau à 150.000 volt absorberait 13 millions. Ce réseau se complète par les postes élévateurs et les postes abaisseurs, qui élèvent à 150.000 volts le courant produit dans les usines, pour le ramener, ensuite, à un voltagc inférieur, au départ des lignes secondaires. Des sous-stations et l'équipement des lignes à électrifier en première étape complètent le programme. Cette première étape comprend environ $1.500 \mathrm{kilo}$ mètres, soit à peu près la moitié du programme envisagé en 1920 Elle s'applique aux lignes subpyrénéennes et à celle de Bordeaux à Irun. En ce qui concerne l'aménagement des vallées de l'Ariège et de la Têt, il n'est prévu que 57.000 francs. Ce travail est aflérent à une seconde étape, pour l'entreprise de laquelle il sera intéressant de connaître les résultats de la première.

- Les dépenses d'établissement, pour l'électrification, sont fort élevées. Leur avantage ne se traduit pas seulement par une économie de charbon ; des économies d'exploitation ont été aussi ascomptées, mais on ne pourra les connaitre avec quelque certitude qu'après la mise en service d'un ensemble de lignes en groupe. L'expériencc sera faite après l'achèvement du programme rle première étape. dont celui de 1923 est une partie. à la coulée.

Ces fours sonl du même modèle que les fuurs Héroult plus petits à revêtement basique, mais la cuve mesure $5 \mathrm{~m}$. 50 de diamètre sur $2^{\mathrm{m}} 50$ de hauteur. L'alimentation est assurée par du triphasé $60 \mathrm{pp}$. sous tension de 90 et $110 \mathrm{~V}$, à la sortie d'un transformateur de $3.000 \mathrm{KVA}$ refroidi dans l'eau, et absorbe normalement de $1.500 \mathrm{a}$ 3.000 KWH.

Industria, 31 décembre 1922.

J. B.

\section{entre deux lignes aériennes parallèles.}

$1^{\circ}$ Pour la ligne japonaise :

$$
C_{11}, C_{22}, C_{33,}, C_{12}, C_{29}, C_{31}
$$

$2^{\circ}$ Pour la ligne américaine :

$$
C_{11}, C_{22}, C_{33},>C_{12}, C_{313}, C_{31}
$$

Notons d'ailleurs qu'aree :

$$
\mathrm{C}_{11}=\mathrm{C}_{12}=\mathrm{C}_{13}=\mathrm{C}_{21}=\mathrm{C}_{22}=\mathrm{C}_{23}=\mathrm{C}_{31}=\mathrm{C}_{32}=\mathrm{C}_{33}
$$

deux installations triphasées n'auraient aucune tendance ì se synchroniser.

L'auteur de l'article a réalisé l'expérience avec deux alternateurs de $5 \mathrm{kVA}$ et des condensateurs téléphoniques.

General Electric Review.

\section{par les Compagnies de chemins de fer en 1923.}

Réseau De P.-O.

La dépense, inférieure à celle prévue pour le Midi, esl de 90 millions. Mais il y a lieu de remarquer qu'en ce qui concerne la création de l'énergie, il y a seulement 6 millions, correspondant à la participation au capital de la Socićté qui construit l'usine d'Eguzon. Par suite de la combinaison spéciale, relative à l'aménagement de la Haute Dordogne,les dépenses de cette dernière opération ne figurent pas aux travaux complémentaires. L'énergie nécessaile pour les lignes comprises au programme de première étape proviendra, d'ailleurs, non seulement des usines de la Haute Dordogne, actionnées par les chutes actuellement en cours d'aménagement, ainsi que de celle d'Eguzon, mais encore du groupe des centrales thermiques de la région parisienne. Toutefois, celles-ci fonctiommeront, surtoul, comme usines de secours ou d'appoint et l'énergie qu'elles fourniront est appelće à diminuer de plus en plus.

On prévoit, pour 1923, l'électrificalion de la ligne Paris-Vierzon.

\section{RESEAU DI L'ETAT}

Le réscau de l'Elat figure pour 45 millions. L'électrilication ne s'y applique qu'à la banlieue de Paris.

La ligne dont la mise en service électrifićc est prévue pour 1923 est celle de Paris à Bécon-les-Bruyères.

$$
\text { Réseau de P.-L.-M. }
$$

Le programme de P.-L.-M, pour 1923, ne comporte que 11 millions et n'est relatif qu'à la ligne de Culoz à Modane, alimentcé par du courant acheté à une société privée. La Compagnie ne compte, en effet, utiliser que de l'énergie provenant de l'industrie, pour la réalisation de la première étape de son programme, qui comprend, outre la ligne Culoz-Modane, la ligne nouvelle de Nice a Coni et la banlieue de Nice, entre Cannes et Menton. 


\section{L'électrification des Chemins de Fer Suédois.}

L'electrilication des lignes de chemins de for est tress artivement entreprise en Suèle, oì les progres accomplis par l'utilisalion de la traction électrique ont ćté rapises el concluants.

la plus inportante des lignes qui utilise actuellement la tracLion ćlectrique est celle de Lulea, sur la mer Ballique, à Riksgriensen, à la frontière norvégienne, el à Narvick, sur l'océan AtlanLique. La partie suédoise de cette ligne a 268 milles de longueur el le service comporte déjà 45 locomotives électriques. Sur beau- coup de ses sections, le trafic esl plus considérable qu'avant la guerre.

Une autre ligne importante, où l'électrification, commencée cette ammée, sera poursuivie très activement, est celle de Stockholm à Gothembourg, qui desservira les principaux embranchements du sud llu pays. Enfin, l'électrification de toutes les lignes est à l'étude, et sa réalisation est envisagée, pour un avenir prochain.

Journée industrielle.

\section{L'électrification des Chemins de Fer Fédéraux est accélérée.}

Le programme d'électrificalion des chemins de fer fédéraux, ćlaboré en 1898, prévoyait que l'on effectuerait chaque année la Lransformation de 100 à 110 kilomètres de voies, de sorte qu'à la fin de 1933 , il y aurait, en chiffres ronds, 1.600 kilomètres exploités à l'électricité. I a longuetr de ligne électrifiée, à l'heure qu'il est, représente 112 kilomètres.

Or, la direction des chemins de fer lédéraux, dans le bul de favòriser le travail dans les industries de la mctallurgie et de la serrurerie, a proposé au Département des chemins de fer d'accélérer l'électrification, de telle sorte que les 1.600 kilomètres prévus soient exécutés en 1928, soit cinc ans plus tôt que ne le prévoyait le pro- 'gramme primitif et, par conséquent, sur la base de 190 kilomètres par année.

La réalisation de ce nouveau programme entrainerait la nécessité d'effectuer, cinq ans plus tôt que ne l'envisageait le projet de 1918 , une dépense de 215 millions de francs.

Une condition de cette exécution serait loctroi, par la Confédération, d'une subvention d'une soixantaine de millions de francs échelonnce sur quatre ou cinc ans.

Le chef du Département des chemins de fer a saisi le Conseil fédéral de ce projet et il a reçu l'autorisation d'élaborer le projet de loi y relatif. Journée Industrielle.

\section{L'exportation de lénergie électrique suédoise au Danemark.}

La Suède et la torvège disposent de plus de houille blanche qu'elles n'en jeuvent utiliser en l'état acluel de leur industrie. Le Danemark, par contre, en est à peu près complètement dépourvu : aussi les deux premiers Etats ont-ils conch avec le troisieme un arrangement aux temes duquel ils s'engagent à lui livrer une partie de leur excédent d'énergie.

Le courant sera naturellement transporté sous haut voltage. Actuellement, la ligne de transmission n'est pas encore construite ; seuls, les plans sont au point, et ils viennent d'être soumis à l'examen d'une commission hydroćlectrique interscandinave, composée, pour la Suède, du colonel Hansen, président du service royal des chutes d'ean suédoises ; pour la Norvège, du directeur général Strevold-Hansen, et pour le Danemark, du professeur Rung. Deux commissions ont été chargées d'étudier ce rapport, l'une au point de vue technique, l'autre sous son aspect économique et juridique, la pose de la ligne de transport devant peut-être entraîner certaines modilications dans le texte des lois qui réglementent actuellement l'exploitation de l'énergie hydroélectrique en Suède et en Norvège.

La Suède exporte déjà de l'énergie en Danemark depuis plusieurs années, par le moyen de câbles sous-marins établis dans le Sund. Dans le cas présent, on avait d'abórd songé à recourir au même procédé, mais des investigations minutieuses ont permis aux ingénieurs de se renclre compte que l'établissement d'une ligne aérienne au-dessus du Sund était une entreprise parfaitement réalisable ; la profondeur moyenne du détroit est faible, et le sol suffisamment résistant peut servir de support à des pylònes de grande hauteur. Le courant sera donc transporté par un circuit aérien. Cette solution permet de transmettre l'énergie sous une haute tension (132.000 volts), alors que dans le cas des câbles sous-marins il est nécessaire d'abaisser le courant, tout le long de la section noyée, à un très bas voltage. La première combinaison est donc plus économique que la seconde, parce qu'elle supprime les abaisseurs et les redresseurs de tension nécessités par la ligne sous-marine.

La question de l'exportation de l'énergie électrique est entrée dans sa phase pratique depuis plusieurs années, et elle n'a, en général, soulevé aucun problème technique ou juridique particulier. Indépendamment de la Suède, la Suisse est également grande exportatrice d'électricité. En 1921, sur un total de 4 millions HP de réserves hydroélectriques, elle exploitait à peine $800.000 \mathrm{HP}$; son exportation durant la même année s'est élevée à 107.768 kilowatts (146.000 HP environn), dont 36.610 allèrent à l'Allemagne, 24.276 à la France et 16.859 à l'Italie.

La Suède, elle, dispose de 6.200.000 HP environ ; elle en exploitait 1.210 .000 à la fin de 1920 ; la Norvège a des réserves presque doubles des précédentes ; elle dispose de $12.300 .000 \mathrm{HP}$. dont elle n'utilise que $1.260 .000 \mathrm{HP}$. Il n'existe pas actuellement de statistique permettant d'établir les quantités d'énergie exportées par ces deux pays dans les Etats voisins.

La Journée Indristrielle.

\section{Les grandes forces hydrauliques de la Bavière.}

L'aménagement des grandes forces hydrauliques de la Bavière, dont le Génie civil a déjà parlé. à plusieurs reprises, fait l'objet, dans la Zeitschrift des Vereines deutscher. Ingenieure, du 28 octobre, d'une note de M. Mattern qui donne des graphiques totalisant les puissances moyennes soit amuelles, soit mensuelles. de chacun des bassins hydrologiques utilisables : l'Inn, l'Isar, la Lech, l' Iller, le Danube, le Main, etc. La puissance mayenne annuclle globale de cet ensemble est évaluée à 1.357 .000 kilowatts.

Outre les usines hydro-ćlectriques déjà en service depuis long: temps, M. Mattern cite celles qui sont en construction : l'usine du lac Walchen, en amont de Munich; les usines sur l'Isar, en amont et en ayal de Munich, les usines de l'Alz; les usines de I'Inn. appartenant à la Compagnie bavaroise d'Aluminium ; l'usine sur la Lech, à Meitingen.

L'auteur, donne des indications générales sur les conditions d'établissement de ces groupes d'usines et sur les cahiers des charges de concession ; la durée des concessions est fixée en principe à 70 ans, avec faculté de rachat après les deux tiers de cette durée. A l'expiration de la concession, les ouvrages hydrauliques et les bàtiments font retour gratuitement à l'Etat, les installations électriques seules étant rachetées par celui-ci. Il expose également des considérations ascez longues sur l'établissement des barrages-réservoirs, et sur les avantages économiques à atteindre de cet aménagement des forces hydrauliques bavaroises.

Journal du Four électrique. 


\section{BIBLIOGRAPHIE}

Les grands réseaux de transport d’énergie électrique à très haute tension. - Compte rendu des travaux de la Conférence internationale des Grands Réseaux de transport d'énergie électrique à très haute tension, tenue à Paris, du 21 au 26 novembre 1921 . - Un rolume de 1.300 pages, avec 350 figures, édité par les soins de M. Tribot-Laspière, Secrétaire général de l'Union des Syndicats de l'Electricité, secrétaire de la Conférence, 25, boulevard Malesherbes, Paris. - Prix, cartonné : $100 \mathrm{fr}$. - En vente à la librairie de la Houille Blanche, 23, Grande-Rue, Grenoble.

On s'étonnera peut-être que le présent volume, relatif à une Conférence tenue en novembre 1921, n'ait paru qu'en octobre 1922.

Si ce délai a été aussi long, c'est d'abord en raison du temps qui a été nécessaire pour l'impression de 1.300 pages et de centaines d'illustrations. ainsi que pour la mise au point de longues traductions. C'est aussi parce que tout le contenu du volume a été communiqué non seulement aux délégués officiels et aux auteurs des rapports, mais aussi à toutes les personnes qui ont pris part aux discussions : ceci, afin d'éviter les erreurs dans toute la mesure du possible et d'offrir un texte présentant le maximum de garanties. Comme beaucoup de ces personnes habitent loin de Paris, et plusieurs trìs loin de l'Europe (quelques-unes aux Etats-Unis et au Japon), il a fallu plusieurs mois pour correspondre avec elles.

Les souscripteurs ont reçu leur exemplaire.

Quant aux personnes qui n'ont pas souscrit et qui désirent recevoir cet important volume, elles sont informées qu'il ne reste actuellement qu'environ 150 exemplaires disponibles et que l'ouvrage ne sera pas réimprimé. Elles sont priées d'adresser leur commande le plus tòt possibłe à l'Union des syndicats de l'Electricité, 25, boulevard Malesherbes, Paris.

Ce compte rendu contient :

$1^{\circ}$ Une notice sur l'objet des travaux de la Conférence ;

20 Un rapport général de $M$. Boucherot;

$3^{\circ}$ Le texte in extenso des 68 rapports présentés à la Conférence ;

$4^{\circ}$ La reproduction sténographique des discussions qui ont suivi chaque rapport.

Ce volume constitue une documentation exceptionnelle, car il contient non seulement des renseignements pratiques sur tout ce qui a été fait dans le monde entier sur la technique des courants à très haute tension, mais aussi l'appréciation des ingénieurs les plus compétents sur ces questions.

Pour tous renseignements ou commandes nos lecteurs sont priés de s'adresser directement à l'T'nion des Syndicats de l'Electricité, 25, boulevard Malesherbes, Paris.

S. V.

Transformateurs statiques de courants alternatifs, par L. BARBILlion, Professeur à l'Université de Grenoble, Directeur de l'Institut Polytechnique. - Volume $16 \times 25$ de 163 pages. 144 figures ou schémas. Vient de paraitre dans la collection rose de la Bibliothèque de l'ingénieur-électricien. - AlbinMichel, éditeur, 22, rue Huyghens, Paris. - Prix net, broché, $15 \mathrm{fr}$. - En vente à la Librairie de la Houille Blanche, J. Rey, 23, Grande-Rue, Grenoble.

Cet important ouvrage consacré uniquement aux transformateurs statiques à courants alternatifs, constitue, après révision, mise au point et notable augmentation des matières, l'une des parties du cours municipal d'électricité indusirielle de l'Université de Grenoble de M. L. Barbillion, cours paru chez Albin Michel, et dont il a été tiré de nombreuses éditions. En raison de l'importance de plus en plus grande, prise par les diverses branches de l'électrotechnique, l'auteur a dû renoncer à une nouvelle publication de ce cours, dont les dimensions eussent été trop considérables.

Dans ce nouvel ouvrage, l'auteur attache une importance exceptionnelle aux perfectionnements apportés dans l'établissement des transformateurs. En particulier, toute la partie construction et essais a fait l'objet d'une révision profonde.

Les transformateurs statiques ont pris, aujourd'hui, unè importance sans égale. Les tensions de production de l'énergie par les alternateurs modernes ne dépassent guire 10.000 à 15.000 vols par cointre les tensions de transport ne cessent de s'clever. Fi France on équipe des lignes pour 120.000 volts, en Amcirique pour 220.000 volts

Le transformateur est le trait d'union entre la station centrate et la ligne de transport : il constitue l'organe peut-p̂tre le plu important des transmissions d'énergie actuelles et. à ce titre seul, il mérite déjà de fairc l'objet d'une étucle exceptionnellemen attentive.

La protection des centrales contre les influences extérieire a fait l'objet de nombreuses études, mais en pratique les avis son très divisés sur l'efficacité des divers appareils employés -; les transformateurs sont les premiers appareils de la centrale qui ont dû supporter les choes, qui se constatent inévitablement sur toule les lignes à haute tension les mieux établies, et les exploitanls en savent quelque chose, par les claquages des bornes, des bobine de sortie et quelquefois entre haute et basse tension. Les construcfeurs ont été amenés à une construction spéciale des transforma teurs modernes. L'auteur a fait ressortir d'une façon particulièrement heureuse tous les perfectionnements qui ont été apportés dan la conception et la construction res transformateurs. Les lecteur trouveront dans cet ouvrage de nombreux renseignements sur lo calcul, les essais et ta construetion de ces importants appareils.

S. $V$

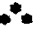

Les grands travaux de France. - Les programmes de recons titution nationale. -1 volume $200 \times 300$, de 192 pages, formant numéro spécial de l'Illustration économique et financière, janvier 1923. - Prix : 5 francs. - En vente à l'Illustration Économiqut et Financière, 35, rue de la Victoire, à Paris.

Ce numéro spécial de l'Illustration Economique et Financior constitue un doçument remarquable, comme permettra de s'en rendre compte la nomenclature des principaux chappitres que nous donnons ci-après :

Il faut produire, par M. Yves Le Trocquer. - La métallurgie et le programme des grands travaux, par M. Robert Pinot. I.'outillage économique de la France el le projet de M. Yves Le Trocquer, ministre des Travaux publics, par M. Albert Lebert. - Le prineipe de la main-d'œuvre allemande; par M. ValetteViallard. - La thèse du Ministère des travaux publics, par M. Jean Maroger.

La Houllle BLANCHE : La production et la distribution de l'énergie électrique en France, par M. 'Tribot-Laspière. - L'aménagement des forces hydrauliques en France, par M. Charles Marquet. - Une région d'avenir, le Gévaudan, par M. Louls Bringer.

Les CHemins DE FER : Lignes nouvelles et travaux supplémentaires (Etat-Est, Paris-Orléans, P.-L.-M.), des Electrification des chemins de fer. Considérations générales Etat, Paris-Orléans, P.-L.-M., Midi.

LES CANAUX ET voIES NAVIGABLES.

S. V.

L'Evidence de la Théorie d'Einstein, par Paul Drumaux, Professeur à l'Université de Gand. -- Un volume in-8 de 72 pages, 1923 ; Broché, 6 francs. - En vente à la Librairie de la Houillt Blanche, 23, Grande-Rue, Grenoble.

C'est une opinion généralement admise et fréquemment exprimée que la théorie d'Einstein est tellement transcendante qu'elle n'est accessible qu'à un petit nombre de privilégiés.

L'auteur se propose de prouver tout le contraire. La théorie de la relativité est devenue synonyme de théorie nitra-savanite, L'auteur entreprend de nous montrer qu'elle relève du bon sens le plus élémentaire.

"La théorie d'Einstein, dit l'auteur en concluant, c'est la guerre aux mots qui ne savent ce qu'ils veulent dire. On a cru nécessairt pour éclairer cette théorie de recourir aux philosophies de Leibnith, 
de Kant et d'autres et d'en chercher des explications jusque dans les nébuleuses spirales. II ne serait peut-être pas inutile d'essayer d'abord de la philosophie de M. Prudhomme. " On est tenté d'ajouter en refermant le livre que la relativité est le retour aux vérités élćmentaires, car on est élonné de voir combien sont évidentes el simples les vérités qui se cachent sous la théorie d'Einstein.

\section{$\because$}

La Force motrice électrique dans l'Industrie, par Eugène Marec, Ancien élève des Ecoles Nationales d'Arts et Métiers, ingénieur diplômé de l'Ecole Supérieure d'Electricité, avec une préface de Paul JANET, Directeur du Laboratoire Central et de l'Ecole supéricure d'Electricitó. - In volume in- $8(25 \times 16)$ de 611 pages avec 541 figures. Prix : 55 francs. - En vente chez GauthierVillars et (ie, 55, quai des Grands-Augustins, à Paris VI ${ }^{\mathrm{e}}$, et à la Librairie Rwy, Grande-Rue, à Grenoble:

L'ingénieur ou l'industriel qui désire se documenter sur l'Electricilé théorique trouve aisćment clans des traités remarquables tels rue ceux de MM. P. Janet, directeur de l'Ecole Supérieure d'Electricité, et Barbillion, directeur de l'Institut Polytechnique de Grenoble, les renseignements dont il a besoin.

Par contre, bien peu d'auteurs se sont occupés en France de guider leurs lecteurs dans l'installation et l'organisation rationnelles des services de force motrice électrique d'usines.

Ce sont ces considérations qui ont conduit M. Marec à élaborer le programme suivant, programme qu'il a réalisé avec un rare bonheur dans son ouvrage.

Prendre le matériel à sa sortie de cher le constructeur;

Faire ressortir ses propriétés caractéristiques, fondamentales en vue d'en permettre le choix raisonni ;

Montrer comment on doit l'installer, l'alimenter, l'entretenir, localiser ses avaries;

Etudier l'organisation générale des services electriques d'une usine ;

Utiliser les connaissances acquises sur les moteurs électriques pour passer une revue critique de leurs principales applications industrielles.

Cet important ouvrage est essentiellement pratiçue. On y trouve des renseignements précicux sur les caractéristiques des différents moteurs ; la question des couples de démarrage est traitéc d'une façon très claire : le nombreux renseignements s'y trouvent sur les questions délicates de dimensionnement des poulies et courroies en fonction des puissances à transmettre.

Un chapitre très complet traile de la question si importante des applications du moteur électrique aux appareils de levage.

Le chapitre réptaration montre que l'auteur est un praticien, qui a mis la main à la pizte, el, chose très rare, l'auteur fait profiter ses lecteurs de toule son expérience personnelle. $I_{z}$ es étudiants, les jeunes dectricions frais imoulu de l'Ecole, les ingénieurs spícialisés ou non, les contremaitres, et môme les ouvriers intelligents trowveront une foule de renseignements pratiques.

L'ouvragr de M. Narec apporte à la littérature technique frangatse une frécieuse contribution. Nous sommes certains qu'il aura un orant succès, aussi bien au bureau d'ćtudes qu'à l'atelier.

Il constitue, à notre avis, un véritable "vade-mecum "des applications modernes de l'Flectricité.

\section{$* *$}

Annuaire du Ministère des Travaux publics (Année 1922). - Un volume in-s de $8 ! 18$ pages. - A. Dumas, éditeur, 6 , rue de la Chaussie-d'Antin, Paris. - Prix broché: $20 \mathrm{fr}$ - - En vente à la I.ibrairie de la Houille Blanche, 2.5, Grande-Rue, Grenoble.

Comme les précéclents, cel Annuaire donne d'abord de nombreux détails sur l'organisation el la constitution de l'Administration centrale des Travaux publics en France ; des renseignements sur les Ecoles des Ponts et Chaussces et des Mines ; la composition de tous les Comités ou Commissions qui relèvent du Ministère des Travaux publies, tels que : le Comité consultatif des chemins de fer ; le Comité consultatif de la navigation intérieure et des ports ; les Commissions des machines à vapeur et des automobiles; la Commission des inventions; la Commission des chaux et ciments; Commission des distributions d'énergie électrique; etc.

L'Annuaire donne l'organisation des Services des Ponts et Chaussées et des Mines dans chaque département, y compris ceux qui faisaient partie de l'Alsace et de la Lorraine et que le Traité de Versailles a rendus à la France.
A signaler aussi, dans les services détachés, la liste des agents du Ministère des Travaux publics affectés aux travaux de reconstitution dans les régions libérées.

La dernière partie comprend les tableaux de classement du personnel du Ministère des Travaux publics : Ponts et Chaussées, Mines et Administration centrale. Parmi ces tableaux il y a lieu de citer ceux qui concernent les ingénieurs des Ponts et Chaussées et des Mines - en congé, hors cadre ou en disponibilité - employés dans les Sociétés industrielles : Compagnies des chemins de fer, tramways, houillères, usines diverses, ateliers de construction, etc.

Le prix de l'Annuaire est de 20 francs pour Paris et de $21 \mathrm{fr} .75$ pour les départements.

Adresser les commandes à: M. A. Dumas, 6, rue de la Chausséed'Antin, Paris, IXe.

Turbines à vapeur, par M. PoIncet, Répétiteur à l'Ecole Polytechnique, professeur à l'Ecole d'application du Génie maritime, 1 vol. gr. in-8 de 340 pages avec 181 figures et une planche inplano. - Broché, 35 fr.; relié souple, 45 fr. (Encyclopédie de Mécanique appliquée, publiée sous la direction de M. L. LEconNu, professeur à l'Ecole Polytechnique, Membre de l'Institut.) J.-B. Baillière et Fils, éditeurs. - En vente à la Librairie de la Houille Blanche, 23, Grande-Rue, Grenoble.

Aménagement hydraulique de la région du Sud-Ouest. - Congrès de Bordeaux. - (17-22 juin 1922). - Rapports, discussions, vœux. - Prix 25 francs. Librairie de l'Enseignement technique, 3, rue Thenard, Paris. - En vente à la Librairie de la Houille Blanche, 23, Grande-Rue, Grenoble.

Le compte rendu de ce Congrès a paru, ainsi d'ailleurs que quelques-uns des rapports qui y ont été lus, dans nombre de Revues techniques. Néanmoins les quelques documents résumés ou disséminés ne sont qu'une bien faible partie de l'ensemble que publie la librairie de l'Enseignement technique, sous le titre ci-dessus. Tous ceux, de quelque région qu'ils soient, et qu'intéresse la question vitale pour la France de l'aménagement et de l'utilisation des cours d'eau, trouveront dans cet ouvrage une étude très approlondie de la question et d'autant plus complète, qu'il s'agit de nombreux rapports émanant tous de personnalités qualifiées pour donner des avis et des directives autorisées.

\section{$*_{* *}^{*}$}

Discours et Mélanges, par Emile PrCand, Secrétaire perpétuel cle l'Académie des sciences. - Gauthier-Villard et $\mathrm{C}^{\mathrm{ie}}$, 55, quai des Augustins, Paris. En vente à la Librairie de la Houille Blanche, 23, Grande-Rue, Grenoble.

Dans cet ouvrage, l'auteur a réuni quelques notices consacrées à la vie et à l'œuvre-de savants éminents, ainsi que des études relatives à l'histoire et à la philosophie des Sciences. On y trouve aussi des articles et conférences se rapportant à divers-sujets qui ont préoccupé l'opinion dans ces dernières années, particulièrement pendant la guerre. Les questions seientifiques devant intervenir de plus en plus dans la vie sociale, les lecteurs trouveront précisément clans cet ouvrage des vues justes sur le ròle de la science et ce qu'on peut attendre d'elle.

\section{$*$}

Note sur le calcul tensoriel. - Astrer de Ponpronas. -- Libraire J. Hermann, 6, rue de la Sorbonne, Paris. Prix $3 \mathrm{fr}$.

\section{$*$}

Organisation administrative industrielle appliquée à la Construction mécanique en petite et moyenne série, de J.-M. CaGnas. Dunod, éditeur, Paris.

Les rayons d'électricité positive et leur application aux analyses chimiques, de Sir J.-J. Thouson, O.-M., R. R. S. (Trad. Fric et Corvity).-J. Hermann, éditeur. - Prix 20 fr.- 225 pages, nombreuses planches. 


\section{INFORMATIONS}

\section{Réseau électrique d'Etat des Régions libérées.}

\section{Ays d'OUTERT'RE DE CONcorres}

Il est ouvert au Ministère des Travaux publics deux concours pour la construction d'un poste de transformation à 120.000 rolts à Laneuveville (Meurthe-et-Moselle), et d'un poste de transformation à 120.000 volts à Landres (Meurthe-et-Moselle).

Pour chacun des concours, les conditions générales du concours sont indiquées dans un devis-programme, auquel sont annexés une notice descriptive des travaux à effectuer, un avant-projet de devis particuliers, et divers plans.

Ces pièces pourront être consultées par les concurrents pendant toute la durée des concours, soit au Ministère des Travaux Publics (Service central des Forces hydrauliques et des Distributions d'énergie électrique, escaliers C ou D, pièce $N^{\circ} 190$ ), soit dans les bureaux de l’Ingénieur du Contròle des Distributions d'Energie Electrique, a. Nancy, les jours de semaine, de 10 heures a midi et de 15 heures à 18 heures.

Les constructeurs qui désirent prendre part aux concours pourront d'ailleurs se procurer ces pieces, soit en venant les retirer au Ministère des Travaux publics, à partir du jour d'ouverture des concours, soit en demandant qu'elles leur soient envoyées ; l'envoi sera fait le jour de louverture des concours.

Les concours, ouverts le 15 janvier 1923 , seront clos le 31 mars 1923.

Les concurrents qui désireraient procéder à des operations sur les terrains des postes devront demander l'autorisation de pénétrer sur ces terrains à M. Le Besnerais, Ingénieur du Contrôle des Distributions d'Fnergie élecrtique à Nancy.

\section{L'Office national des recherches scientifiques et industrielles et des inventions.}

Une loi promulguée au Journal Officiel du 30 décembre dernier a réalisé la transformation de l'Office national des Recherches scientifiques et industrielles et des Inventions en un Office national du même nom, pourvu de la personnalité civile et de l'autonomie financière.

Le décret prévu par cette loi et fixant la constitution et le fonctionnement de ce nouvel office vient lui-même de paraitre. II fixe, avec les attributions de cet organisme de recherches et d'inventions, le mode de constitution de son Conseil national et de son Conseil d'administration.

Le Conseil d'administration, qui vient d'être constitué par lo Ministre de l'Instruction publique, contient les plus grands noms de la science et de l'industrie de notre pays et donne toute garantie sur le fonctionnement et l'avenir de l'Office National des Recherches et Inventions.

\section{Société des Etablissements Beccat.}

Il vient d'être fait concession à cette Société d'une chute du torrent Le Borne pour l'établissement d'une usine hydroćlectrique à Saint-Pierre-de-Rumilly (Haute-Savoie).

La puissance maximum brute de la chute concédée est évaluée à six mille kilowatts, ce qui correspond, compte tenu du rendement normal des appareils d'utilisation, à une puissance maximum disponible de quatre mille cing cents kilowatts. La puissance normale brute est évaluée à quatre mille kilowatts, ce qui correspond à une puissance normale disponible de trois mille kilowatts.

I'entreprise a pour objet principal la lourniture d'énergie i laciérie électrique appartenant au concessionnaire el situce Saint-Pierre-de-Rumilly.

\section{NOTE DE LA RÉDACTION}

Nous recevons des Aleliers de Constructions électriques de belle la lettre que nous reproduisons ci-après :

$$
\text { "Wonsieur, }
$$

"Nous relevons dans l'article : "Inauguration de la ligne Pau-Lourdes, de la Compagnie des Chemins de fer du Midi, électrifiée à 1.500 volts, couranl continu ", paru dans le numéro de novembre-décembre de La Houille Blanche, le passage suivanl:

"Le courant à 1.500 volts continu était fourni par deux sous" stations, l'une à Coarraze-Nay équipée au moyen de commu" tatrices, l'autre à Lourdes équipée à l'aide de convertisseurs à "vapeur de mercure à grand débit. Le Ministre s'est arrêté " devant"ces deux sous-stations et les a visitées en détail. Il a " été reçu par M. Ferrand, Président du Conseil d'administration " et par M. Schwarberg, Directeur général de la Compagnie "Electro-Mécanique, Société qui a équipé complètemenl ces " sous-stations."

"Nous nous permettons de vous faire remarquer que cette relation est en partie erronée : la sous-station çxtérieure de
Coarmaz-Nay a été, en elfet, entièrement éfuipéc avec du matériel de notre construction (1), exception laite, foulctos des transformateurs.

"Comme nous atlachons la plus grande importance à ce détail qui, vous le concevez, constilue une excellente référence en notri faveur, nous vous prions de bien vouloir faire loule rectilication utile dans le prochain numéro de votre Iievue.

"Nous vous prions d'agréer, Monsieur, l'assurance de notte consideration la plus distinguce.

"Le Lirecteur commercial,

$$
\text { "J. B....." }
$$

(1) Disjoncteurs et sectionneurs à 70.000 volts. 